\title{
Prediction of sound power radiated from oil hydraulic pump using FEM and BEM
}

\author{
Toru Yamazaki* and Eiichi Kojima ${ }^{\dagger}$ \\ Faculty of Mechanical Engineering, Kanagawa University, \\ 3-27-1, Rokkakubashi, Kanagawa-ku, Yokohama, 221-8686 Japan
}

(Received 30 October 2002, Accepted for publication 19 November 2002)

Keywords: Vibration, Noise, Fluid power system, Finite Element Method, Boundary Element Method PACS number: 43.40.- r

\section{Introduction}

Improvement of the valve plate design is one of the measures to reduce the noise radiated from an oil hydraulic pump. However, the countermeasure of exciting source alone like this has its limit. To design the quieter pump efficiently by making use of also the vibration control technique, development of a simulation technique is required including the properties of the vibration transmission paths and of the sound radiation mechanism from the pump casing surface. We have already developed the simulation technique using FEM [1-3], which enables one to predict the structural vibration of an oil-hydraulic pump casing under real operating conditions and then to predict the sound power using the experimentally determined sound radiation efficiency. This paper proposes the technique capable of predicting the sound power radiated from the pump by the numerical analysis only using BEM and FEM. It is shown that the radiated sound power levels simulated by the proposed technique agree well with the measured results. It is also demonstrated that our technique is very useful to predict the effect of the improvement of the valve plate design at the early design state.

\section{Modeling}

\subsection{Test pump}

A variable-displacement, swash-plate type of axial piston pump is tested. Specification of the test pump is as follows; number of piston: 9 , displacement: $23 \mathrm{ml} / \mathrm{rev}$, rated pressure: 17.2 MPa, rated speed: $1,450 \mathrm{rpm}$. Figure 1 shows the FEM model of the test pump including a commercially used L-type bracket, which consists of 2,930 hexa-solid elements and 4,936 nodes.

2.2. Replacement of real exciting force and moments with virtual exciting forces

The transient pressure fluctuation in cylinder exerts the force and moments $\left(F_{x}, M_{y}, M_{z}\right)$ on both a swash-plate and end-cover. These real force and moments can be expressed using the pressure fluctuation in cylinder $P_{\mathrm{c}}(\theta)$ by

$$
\begin{aligned}
F_{x} & =\sum_{i=1}^{z} \frac{A_{\mathrm{c}} P_{\mathrm{c}}\left(\theta_{i}\right)}{\cos \alpha}, \\
M_{y} & =\sum_{i=1}^{z} \frac{A_{\mathrm{c}} P_{\mathrm{c}}\left(\theta_{i}\right)}{\cos \alpha}\left(\frac{R \cos \theta_{i}+e}{\cos \alpha}+l \cdot \tan \alpha\right),
\end{aligned}
$$

\footnotetext{
*e-mail: toru@cc.kanagawa-u.ac.jp

†e-mail: kojime01@kanagawa-u.ac.jp
}

$$
M_{z}=\sum_{i=1}^{z} \frac{A_{\mathrm{c}} P_{\mathrm{c}}\left(\theta_{i}\right)}{\cos \alpha} R \sin \theta_{i},
$$

where $A_{\mathrm{c}}$ is a cross-sectional area of cylinder, $\alpha$ is an inclination angle of swash-plate, $z$ is the number of pistons, $R$ is a radius of pitch circle of cylinder, $e$ is an eccentric distance between centers of driving shaft and trunnion axis and $l$ is a normal distance from a center of trunnion axis to a sliding surface of swash-plate.

It can be seen from Eqs. (1)-(3) that the point of application of $F_{x}$ draws a letterform of 8 as shown in Fig. 2. We have to replace this moving exciting force $F_{x}$ with the stationary exciting forces applied on the several node points of the FEM model in order to make the forced vibration analysis by FEM possible. Real moving exciting force can be replaced physically equivalently with the three virtual exciting forces $\left(F_{x .1}{ }^{*}, F_{x .2}{ }^{*}, F_{x .3}{ }^{*}\right)$ applied respectively on the node $\mathrm{A}, \mathrm{B}$ and $\mathrm{C}$ shown in Fig. 2, which are simply determined from the following equations.

$$
\begin{aligned}
& F_{x .1}{ }^{*}=F_{x}, \\
& F_{x .2}{ }^{*}=M_{y} / z_{0}, \\
& F_{x .3}{ }^{*}=\left(F_{x .1}{ }^{*} y_{0}-M_{z}\right) / 2 y_{0} .
\end{aligned}
$$

\subsection{Results of FEM analysis}

The harmonic amplitudes of casing surface vibration were calculated by the forced vibration analysis in FEM code (ANSYS) under the operating condition of pump speed being $1,500 \mathrm{rpm}$ and system pressure $14.0 \mathrm{MPa}$. The calculation is carried out at every harmonic frequency from $225 \mathrm{~Hz}$ of first order to $3,150 \mathrm{~Hz}$ of $14 \mathrm{th}$ order. The harmonic components of the virtual forces at each frequency were calculated from the Fourier transformation of $F_{x}$ obtained using the measured pressure fluctuations in cylinder. We also measured the surface vibrations at 164 node points $(3 \times 164=492)$ so as to verify the accuracy of the FEM analysis.

As can be seen from Fig. 3 showing an example of amplitude spectrum of out-of-plane vibration of pump casing under real operating condition, the simulated result agrees relatively well with the measured result for its complexity. Figure 4 shows the comparison of vibrating shapes simulated and measured at $2,700 \mathrm{~Hz}$ (12th order). The out-of-plane vibration of vertical panel of a bracket and the respiration vibration of abdomen of a pump structure are evidence in both the simulations and the measurements. 


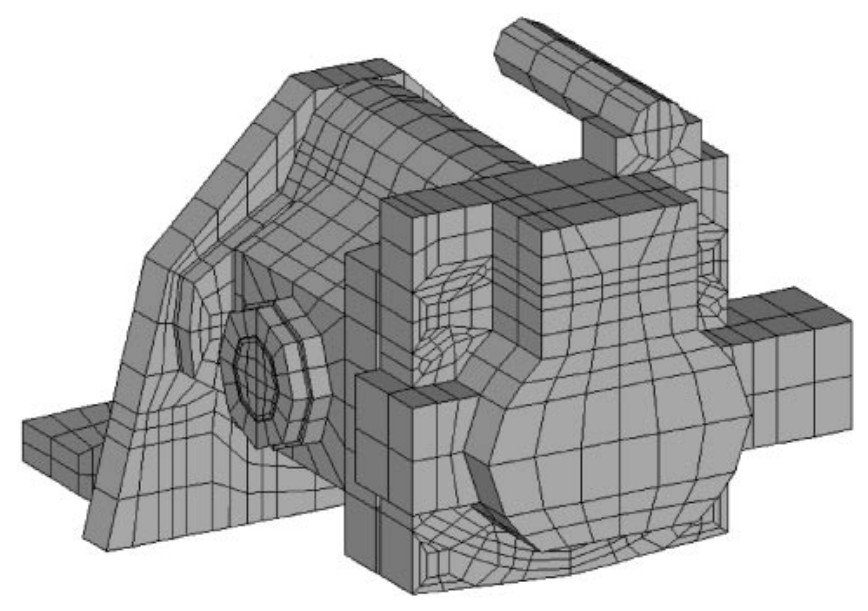

Fig. 1 FEM model of test pump.

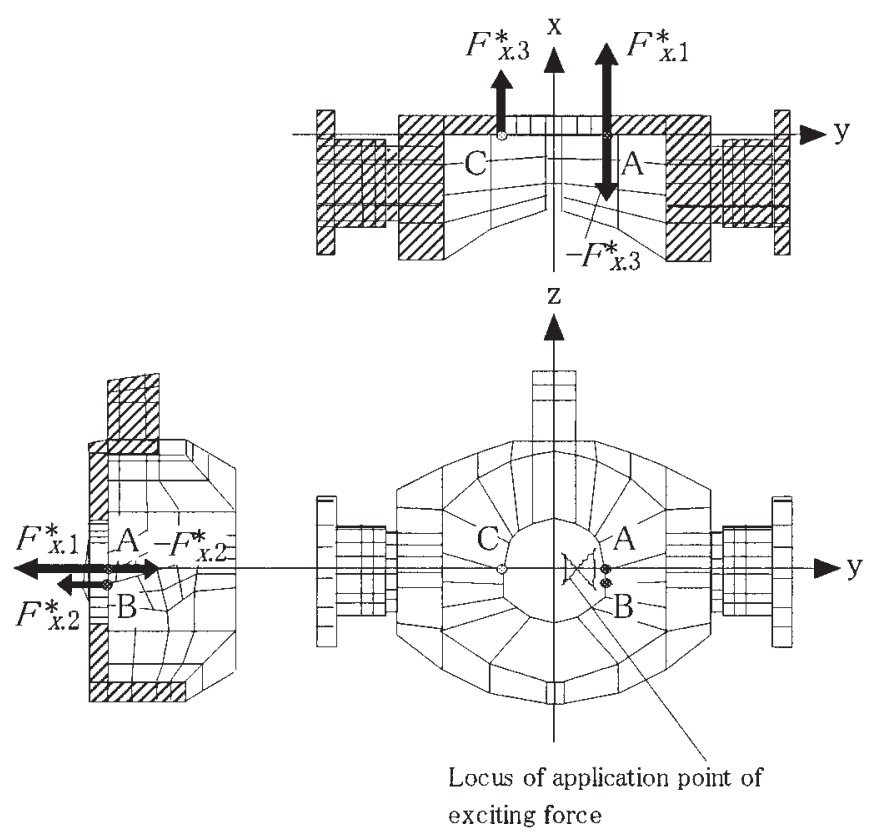

Fig. 2 Virtual forces at point A, B and C.

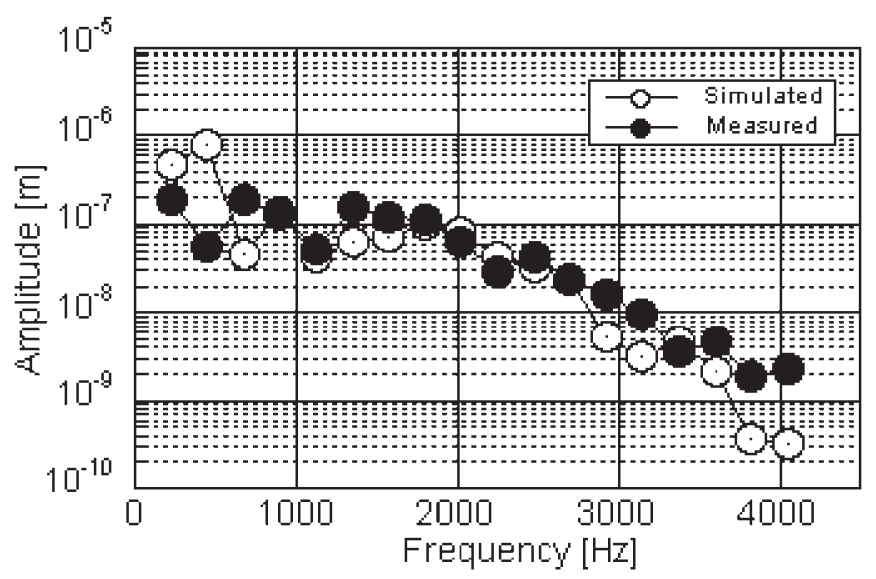

Fig. 3 Measured and estimated vibration level on casing surface.
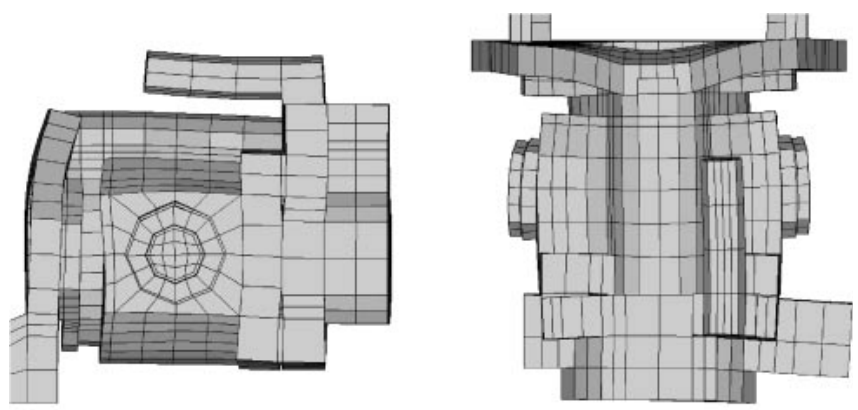

(a) Simulated.
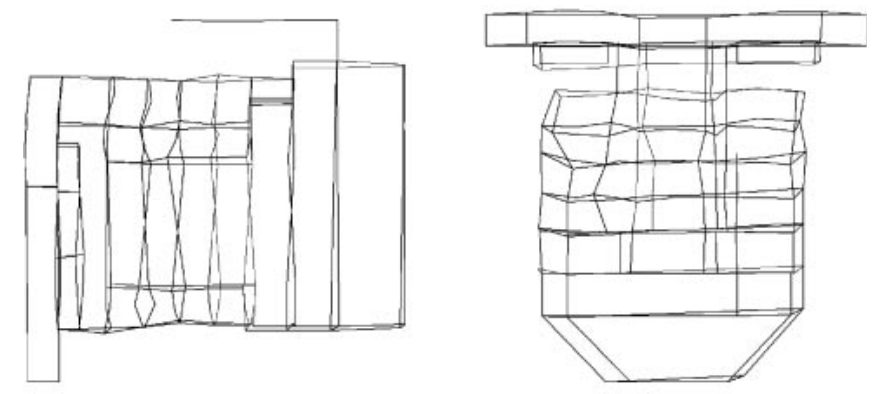

(b) Measured.

Fig. 4 Vibration shape of pump structure.

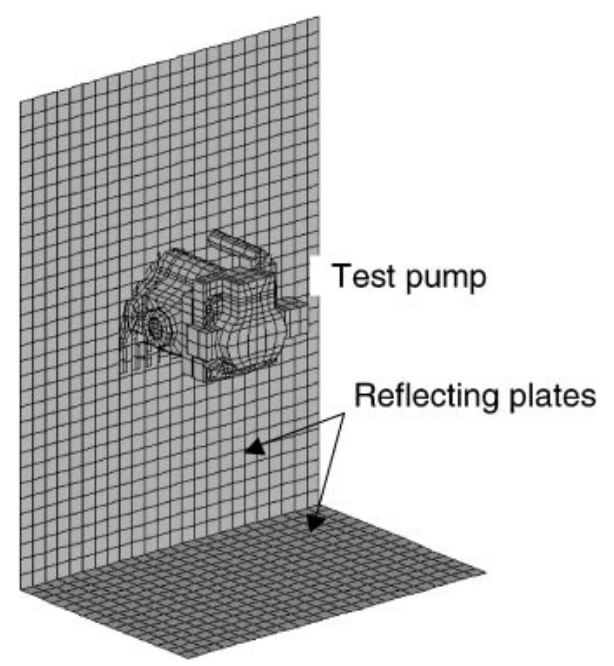

Fig. 5 BEM model.

\section{Analysis of radiated sound power by BEM}

The radiated sound power from the pump was calculated using BEM code (LMS/SYSNOISE). The FEM results were used as the boundary conditions on BEM model. The BEM model of the test pump including the sound fields is shown as Fig. 5. This model includes the reflecting plates, which are used to measure the radiated sound power by intensity method. The BEM model for the pump is made by coarsening the FEM model. The casing vibration displacements obtained in advance by FEM analysis were imported as the boundary conditions for BEM analysis, while the vibration velocities of the reflecting plates were all set to 0 . To determine experimentally the radiated sound power under the real 


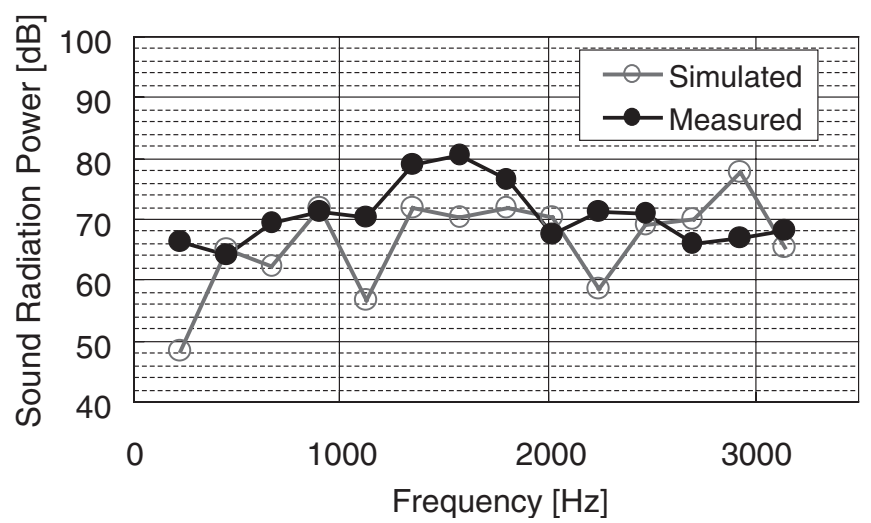

Fig. 6 Simulated and measured sound radiated power.

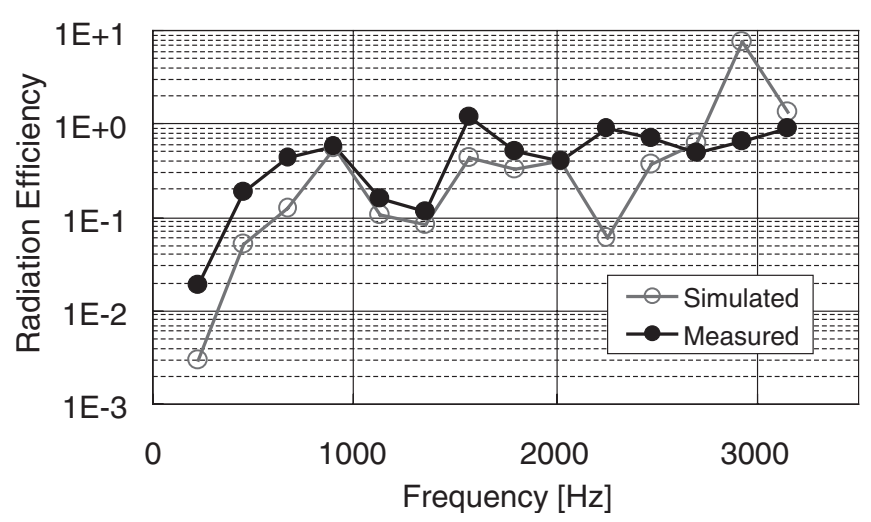

Fig. 7 Sound radiation efficiency.

operation, the sound intensities were also measured on the four measuring planes by using sound intensity scanning method based on ISO9614 Part 2. The sound intensities on the same observe fields as the measuring planes are calculated by BEM. The vibration velocities of pump casing were also measured at selected 190 locations to identify the sound radiation efficiency experimentally.

Figure 6 shows the comparison of the simulated sound power from BEM with the measured results. Both powers agree with a sufficient accuracy for practical usage for its complexity. The calculated radiation efficiencies from BEM also agree well with the measured results as shown in Fig. 7. There are, however, some differences between them because of the error in FEM modeling and the influence of sound radiated from the inlet and outlet pipes close to the pump.

\section{Simulation of sound reduction by improvement of valve plate design}

The sound radiated from the pump under the real operation can be estimated by using FEM and BEM without using any experimental data as shown in Sec. 3. In this paper, effect of the sound reduction by improving the valve plate design was investigated using the above technique. Figure 8 shows two valve plate tested with or without the relief

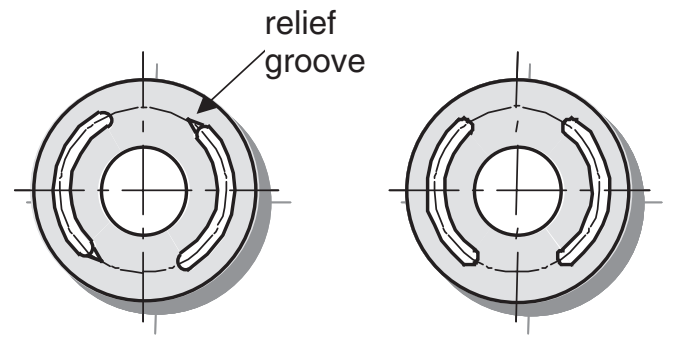

(a) With relief grooves.

(b) Without relief groove.

Fig. 8 Shapes of valve plates.

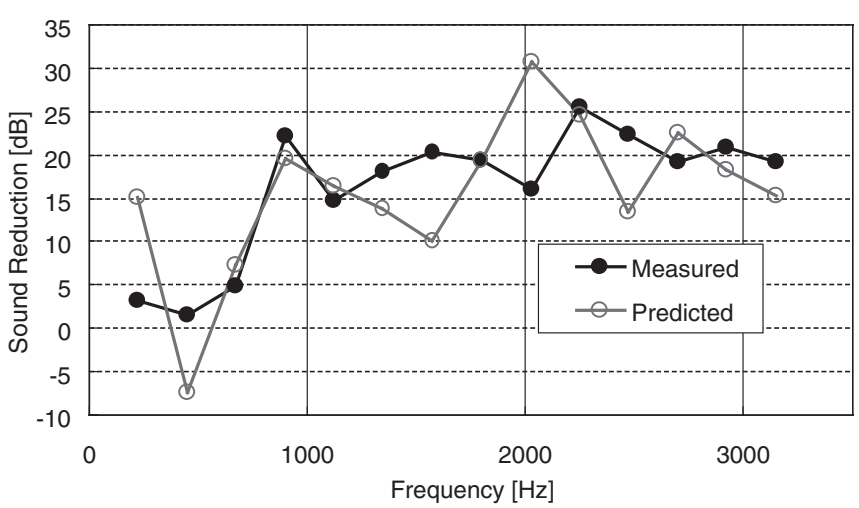

Fig. 9 Comparison of differences between measured and simulated results.

grooves and pre-compression (and pre-expansion) section.

The sound reduction level simulated is shown in Fig. 9 together with the measured level. The overall-value of reduction levels simulated is $15.6 \mathrm{~dB}$, which is close to the measured levels $16.2 \mathrm{~dB}$. In conclusion, our proposed simulation technique is found to be very useful design tool for developing the quieter pump.

\section{Conclusions}

We proposed the simulation technique which predicted the sound power radiated from oil-hydraulic pump under the real operating conditions by numerical analysis alone using FEM and BEM. The simulated results agreed with the measured results with a sufficient accuracy for practical usage. Our proposed simulation technique was verified to be very useful tool at early design stage.

\section{References}

[1] E. Kojima, H. Iwata, N. Hyodo, S. Matsusima and K. Watanabe, "Modal analysis of the structural vibration of an oil-hydraulic pump," Trans. JFPS, 27(2), 315-322 (1996).

[2] E. Kojima, S. Matsusima, K. Watanabe and S. Shimizu, "FEM analysis of structural vibration of an oil-hydraulic pump under real operating conditions," Trans. JFPS, 29(3), 79-86 (1998).

[3] S. Shimizu and E. Kojima, "Estimation of sound radiation power of hydraulic fluid power pump by FEM analysis," Trans. JFPS, 30(9), 163-169 (1999). 